\title{
QUANTITATIVE JUMP THEOREM
}

\author{
P. I. KALEDA
}

\begin{abstract}
The jump theorem proved by Mishchenko and Pontryagin more than fifty years ago is one of the fundamental results in the theory of relaxation oscillations. Its statement is asymptotic in character. In this paper we present a quantitative analogue of it. This means the following. The jump theorem describes the map along trajectories (the Poincaré map) from a transversal 'before the jump' to a transversal 'after the jump'. This map is exponentially contracting, and its deviation from the jump point with respect to the slow coordinate is of order $\varepsilon^{2 / 3}$, where $\varepsilon$ is the small parameter in the fast-slow system. These estimates are asymptotic. Normalizing the system by choosing the scale, we prove that for all $\varepsilon$ no greater than $e^{-12}$, the Poincaré map is defined, its deviation lies in the interval $\varepsilon^{2 / 3}\left[e^{-6}, e^{3}\right]$, and the map itself is a contraction with a coefficient that does not exceed $e^{-k(\varepsilon)}$, where $k(\varepsilon) \geq$ $1 /(6 \varepsilon)-10^{3}$. The main tool used in the investigation is the method of blowup with different weights, in the form described by Krupa and Szmolyan.
\end{abstract}

\section{§1. Introduction: The Classical Jump theorem AND its 'REFinement'}

We consider a singularly perturbed system

$$
\begin{aligned}
\varepsilon \frac{d x}{d \tau} & =F(x, y, \varepsilon), \\
\frac{d y}{d \tau} & =G(x, y, \varepsilon),
\end{aligned} \quad x \in \mathbb{R}^{n}, y \in \mathbb{R}^{m}, F, G \in C^{k}, k \geq 3,
$$

or, after passing to the fast time (making the change of variables $t:=\tau / \varepsilon$ ),

$$
\begin{aligned}
& \dot{x}=F(x, y, \varepsilon), \\
& \dot{y}=\varepsilon G(x, y, \varepsilon) .
\end{aligned}
$$

The well-known phenomenon of relaxation oscillations consists in the following. For a small value of the parameter, for some time a trajectory of the system (11) slowly (together with the parameter) drifts along the slow surface, that is, the set $\{F(x, y, 0)=0\}$. Then, on reaching a certain critical point, a so-called jump point where the slow surface is tangent to the direction of the $x$-axis, the trajectory quickly (the speed of motion is asymptotically independent of the parameter) jumps to another part of the slow surface, then slowly drifts to the next jump point, and so on.

The transition from slow motion to fast is called a jump, and it is described by the well-known jump theorem (see, for example, the survey [1]). Proved by Pontryagin and Mishchenko [4] more than fifty years ago, the jump theorem is one of the fundamental results of the theory of relaxation oscillations. For the planar case, a simpler proof of this

2010 Mathematics Subject Classification. Primary 34E15; Secondary 34C26, 34E05, 34E20, 37C10, $37 \mathrm{G} 10$.

Key words and phrases. Relaxation oscillations, fast-slow system, jump point, resolution of singularities, normal form.

This research was supported by the Russian Foundation for Basic Research (grant no. 7-01-00017-a) and by RFBR/CNRS (grant no. 05-01-02801-CNRSa). 
theorem was obtained later by Krupa and Szmolyan [8], using the technique developed by Dumortier and Roussarie [6].

We state the jump theorem in the version given by Krupa and Szmolyan 8. Consider the system (11) with one-dimensional phase variables and parameter. Suppose that the origin is a jump point:

$$
F(0,0,0)=0, \quad \partial F(0,0,0) / \partial x=0 .
$$

Suppose that the following nondegeneracy conditions hold:

$$
\frac{\partial^{2} F}{\partial x^{2}}(0,0,0) \neq 0, \quad \frac{\partial F}{\partial y}(0,0,0) \neq 0, \quad G(0,0,0) \neq 0 .
$$

Under these conditions we can assume without loss of generality that

$$
\frac{\partial^{2} F}{\partial x^{2}}(0,0,0)>0, \quad \frac{\partial F}{\partial y}(0,0,0)<0, \quad G(0,0,0)<0 .
$$

Now, under the assumption that (2) holds, using a change of the coordinates and time we can reduce the system (11) to the following form:

$$
\begin{aligned}
& \dot{x}=-y+x^{2}+h(x, y, \varepsilon), \\
& \dot{y}=\varepsilon(-1+g(x, y, \varepsilon)),
\end{aligned}
$$

where $h(x, y, \varepsilon)=O(x, y, \varepsilon)$ and $g(x, y, \varepsilon)=O(x, y, \varepsilon)$. The slow surface for this system is the parabola $y=x^{2}$.

Suppose that $\rho>0$ is small. Consider the set

$$
\Delta^{\text {in }}:=\left\{\left(x, \rho^{2}\right): x \in J\right\},
$$

where $J$ is an interval of small length such that $\Delta^{\text {in }}$ is transverse to the phase flow of the system (3) for small $\varepsilon>0$ and intersects the branch $S_{a}$ of the slow surface corresponding to the inequality $x<0$. Furthermore, we introduce the notation

$$
\Delta^{\text {out }}:=\{(\rho, y): y \in \mathbb{R}\} .
$$

Theorem 1 (Jump theorem; see [8]). There exist numbers $\rho>0$ and $\varepsilon_{0}>0$ for the system (3) such that for any $\varepsilon \in\left(0, \varepsilon_{0}\right]$,

- the recurrence map $\pi_{\varepsilon}: \Delta^{\text {in }} \rightarrow \Delta^{\text {out }}$ is defined;

- the trajectory $S_{a, \varepsilon}$ intersecting the transversal $\Delta^{\text {in }}$ at a point $\left(a, \rho^{2}\right)$ intersects the transversal $\Delta^{\text {in }}$ at the point $(\rho, \eta(\varepsilon))$, where

$$
\eta(\varepsilon)=O\left(\varepsilon^{2 / 3}\right)<0
$$

- the map $\pi_{\varepsilon}$ is a contraction, and

$$
\left(\pi_{\varepsilon}\right)^{\prime}=O\left(e^{-c / \varepsilon}\right)
$$

for some $c>0$.

However, this theorem only gives the asymptotic behavior, and this often turns out to be insufficient for applications. For example, is $\varepsilon_{0}=10^{-1}$ sufficiently small so that the map $\pi_{\varepsilon}$ at least exists? Or what about $\varepsilon_{0}=10^{-1000}$ ? Theorem 1 does not give an answer to this type of question. The same can be said about the other parameters in the asymptotic formulae.

The main goal of this paper is to give a quantitative analogue of Theorem 1, that is, to determine the smallness of the parameter concretely, and give concrete estimates for the asymptotic formulae (4) and (5), which we write down explicitly. The precise statement of this result is contained in Theorem 6. The main method we use in the proof is the method of blowup at a singular point with different weights, borrowed from [8]. 
Furthermore, in the course of proving the main result, the author obtained another result, which follows. In a sense, it refines Theorem 1. To state it (Theorem 4) we shall need the following definition.

Definition 2. An invariant manifold of the system (11) that is $O(\varepsilon)$-close to the slow surface outside a neighbourhood of a jump point is called a true slow surface (in the two-dimensional case, a true slow curve).

Remark 3. The existence of a true slow surface is well known (see, for example, [7]); it may not be unique.

Theorem 4. Consider the system (31) and a smooth family $\left\{S_{\varepsilon}\right\}$ of true slow curves. Then there exists $\varepsilon_{0}>0$ such that the family $\left\{S_{\varepsilon}\right\}$ describes a curve $\varepsilon=\eta(y)$ on the transversal $\Delta^{\text {out }} \times\left(0, \varepsilon_{0}\right)$ which has a (one-sided) derivative at zero.

Note that the smoothness of $\eta$ outside a neighbourhood of zero is obvious from the general theory of ODEs; it follows from Theorem 1 that if the one-sided derivative at zero $\eta^{\prime}\left(0^{-}\right)$exists, then it is equal to zero.

The author would like to express his gratitude to his beloved teacher, Professor Yuliu Sergeevich Il'yashenko, for posing the problem and for his constant support during this work, as well as to Il'ya Shchurov for reading the manuscript and for his valuable remarks concerning it.

\section{$\S 2$. The STatement of The Quantitative Jump Theorem}

In the $(x, y)$-plane we consider the rectangle $U$ with sides parallel to the coordinate axes and with vertices $(-13 / 12,-1),(1,1)$ (see Figure 1). We consider a smooth system of the form

$$
\left\{\begin{array}{l}
\dot{x}=F(x, y, \varepsilon), \\
\dot{y}=\varepsilon G(x, y, \varepsilon),
\end{array}\right.
$$

and suppose that

$$
\begin{aligned}
& F(x, y, \varepsilon)=\left(x^{2}-y\right)(1+h(x, y, \varepsilon)), \\
& G(x, y, \varepsilon)=-1+g(x, y, \varepsilon),
\end{aligned}
$$

where

$$
h(x, y, \varepsilon)=O(x, y, \varepsilon), \quad g(x, y, \varepsilon)=O(x, y, \varepsilon) .
$$

Note that for any system of the form (6) satisfying conditions (2) we can choose coordinates such that its right-hand sides have the form (7).

The system (6) has a jump at the origin.

Using a change of coordinates of the form $(x, y, \varepsilon) \rightarrow\left(k x, k^{2} y, k^{3} \varepsilon\right)$ it is always possible to make sure that for all $(x, y, \varepsilon) \in U \times\left[0, \varepsilon_{0}\right], \varepsilon_{0}=e^{-3}$, the following conditions hold for the functions $h$ and $g$ :

$$
\begin{aligned}
& |h(x, y, \varepsilon)| \leq 1 / 2, \\
& |g(x, y, \varepsilon)| \leq 1 / 2
\end{aligned}
$$

and for their gradients:

$$
\begin{aligned}
\|\operatorname{grad} h\|_{1} & \leq 10^{-2}, \\
\|\operatorname{grad} g\|_{1} & \leq 10^{-2},
\end{aligned}
$$

where $\left\|\left(\xi_{1}, \xi_{2}, \xi_{3}\right)\right\|_{1}:=\sum_{1}^{3}\left|\xi_{i}\right|$. The phase portrait of this system is depicted in Figure 1 . 


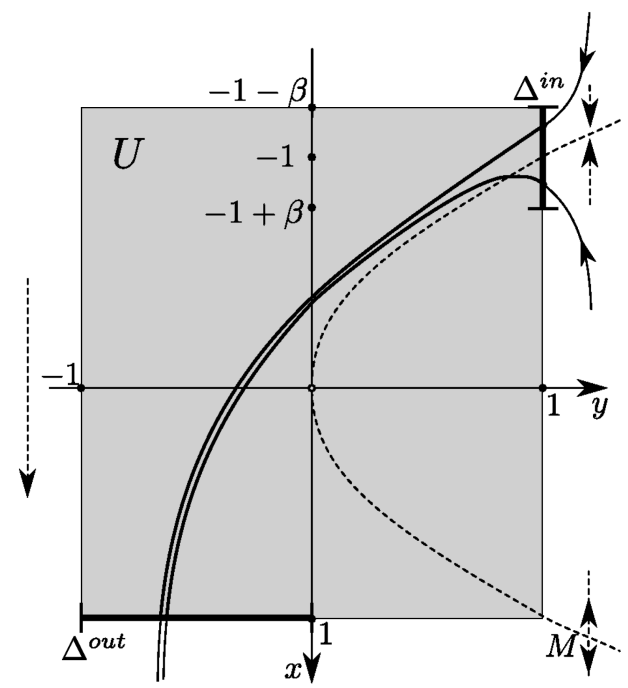

Figure 1. Phase portrait of the system (6) for small $\varepsilon>0$

Remark 5. The system (6) with the right-hand sides (7) is the normal form of the fastslow system. The normalization consists in normalizing the slow curve $\left(x^{2}-y=0\right)$ with the consequent change of scale that the system imposes on itself (the requirements (9) and (10) ).

We consider the 'entrance' transversal

$$
\Delta^{\text {in }}:=\{(x, y): x \in(-1-\beta ;-1+\beta), y=1\}, \quad \beta=1 / 12,
$$

and the 'exit' transversal

$$
\Delta^{\text {out }}:=\{(x, y): x=1, y \in(-1 ; 0)\} .
$$

Theorem 6 (Quantitative jump theorem). Suppose that conditions (9) and (10) hold. Then for any $\varepsilon \in\left(0, \varepsilon_{0}=e^{-12}\right)$ the system (6) satisfies:

(1) the map along trajectories $\pi_{\varepsilon}: \Delta^{\text {in }} \rightarrow \Delta^{\text {out }}$ exists;

(2) if $p \in \Delta^{\text {in }}$, then $\pi_{\varepsilon}(p)=(1, \nu(\varepsilon))$, and $-e^{3} \cdot \varepsilon^{2 / 3} \leq \nu(\varepsilon) \leq-e^{-6} \cdot \varepsilon^{2 / 3}$;

(3) the map $\pi_{\varepsilon}: \Delta^{\text {in }} \rightarrow \Delta^{\text {out }}$ is a contraction, and $\left(\pi_{\varepsilon}\right)^{\prime} \leq e^{-\left(1 /(6 \varepsilon)-10^{3}\right)}$.

\section{$\S 3$. Blowup, the MAIN TOOL OF THE PROOF}

We consider the extended system

$$
\left\{\begin{array}{l}
\dot{x}=F(x, y, \varepsilon), \\
\dot{y}=\varepsilon G(x, y, \varepsilon), \\
\dot{\varepsilon}=0 .
\end{array}\right.
$$

We apply the method of blowup to this system, similarly to the proof of the jump theorem in 8 . As a result, the jump point (the origin) 'blowsup' to a two-dimensional sphere. In certain directions transverse to the sphere, we obtain hyperbolicity, which enables us to conduct a complete analysis by standard methods.

Let $B:=\mathbb{S}^{2} \times[0,1]$. The blow-up transformation is regarded as a map

$$
\Phi: B \rightarrow \mathbb{R}^{3}
$$

with $(\bar{x}, \bar{y}, \bar{\varepsilon}) \in \mathbb{S}^{2}$. 
The blow-up transformation takes the following form:

$$
x=\bar{r} \cdot \bar{x}, \quad y=\bar{r}^{2} \bar{y}, \quad \varepsilon=\bar{r}^{3} \bar{\varepsilon} .
$$

Let $X$ denote the vector field (11). Since the vector field $X$ vanishes at the point $(0,0,0)$, there exists a vector field $\bar{X}$ on $B$ such that $\Phi_{*}(\bar{X})=X$, where $\Phi_{*}$ is induced by $\Phi$. Finally, it remains to analyse the vector field $\bar{X}$ on $B$. This is sufficient, since $\Phi(B)$ is a full neighbourhood of zero.

For our analysis in the domain $B$ we shall use the charts $K_{1}:=\{\bar{y}=1\}, K_{2}:=\{\bar{\varepsilon}=1\}$, and $K_{3}:=\{\bar{x}=1\}$. The chart $K_{2}$ describes the upper half-sphere $\bar{\varepsilon}>0$, and the charts $K_{1}$ and $K_{3}$ describe neighbourhoods of two parts of the equator that are required for the analysis.

The blow-up transformation in the charts $K_{j}$ takes the form:

$$
\begin{array}{lll}
x=r_{1} x_{1}, & y=r_{1}^{2}, & \varepsilon=r_{1}^{3} \varepsilon_{1} ; \\
x=r_{2} x_{2}, & y=r_{2}^{2} y_{2}, & \varepsilon=r_{2}^{3} ; \\
x=r_{3}, & y=r_{3}^{2} y_{3}, & \varepsilon=r_{3}^{3} \varepsilon_{3} .
\end{array}
$$

Remark 7 . Henceforth we shall use the following notation. If $P$ is some object from the original problem (11), then we denote the same object after the blow-up transformation by $\bar{P}$, and the same object in the chart $K_{j}, j=1,2,3$ by $P_{j}$.

Let $\kappa_{i j}$ denote the transition function from the chart $K_{i}$ to the chart $K_{j}$. The following lemma is proved by a simple check.

Lemma 8 ([8, Lemma 2.2]). The following formulae hold:

- for $\kappa_{12}$ :

$$
x_{2}=x_{1} \varepsilon_{1}^{-1 / 3}, \quad y_{2}=\varepsilon_{1}^{-2 / 3}, \quad r_{2}=r_{1} \varepsilon_{1}^{1 / 3} \quad \text { for } \varepsilon_{1}>0
$$

- for $\kappa_{12}^{-1}$ :

$$
x_{1}=x_{2} y_{2}^{-1 / 2}, \quad r_{1}=r_{2} y_{2}^{1 / 2}, \quad \varepsilon_{1}=y_{2}^{-3 / 2} \text { for } y_{2}>0
$$

- for $\kappa_{23}$ :

$$
r_{3}=r_{2} x_{2}, \quad y_{3}=y_{2} x_{2}^{-2}, \quad \varepsilon_{3}=x_{2}^{-3} \quad \text { for } x_{2}>0
$$

- for $\kappa_{23}^{-1}$ :

$$
x_{2}=\varepsilon_{3}^{-1 / 3}, \quad y_{2}=y_{3} \varepsilon_{3}^{-2 / 3}, \quad r_{2}=r_{3} \varepsilon_{3}^{1 / 3} \text { for } \varepsilon_{3}>0 .
$$

$\S 4$. Dynamics in the chart $K_{1}$ : The map 'Before the Jump'

We substitute (13) into the system (11), perform the change of time variable $t=r_{1} \tau$, and obtain

$$
\left\{\begin{aligned}
x_{1}^{\prime} & =\left(x_{1}^{2}-1\right)\left(1+h_{1}\right)-\frac{1}{2} x_{1} \varepsilon_{1}\left(g_{1}-1\right), \\
r_{1}^{\prime} & =\frac{1}{2} r_{1} \varepsilon_{1}\left(g_{1}-1\right), \\
\varepsilon_{1}^{\prime} & =-\frac{3}{2} \varepsilon_{1}^{2}\left(g_{1}-1\right),
\end{aligned}\right.
$$

where $g_{1}\left(x_{1}, r_{1}, \varepsilon_{1}\right):=g\left(r_{1} x_{1}, r_{1}^{2}, r_{1}^{3} \varepsilon_{1}\right)$ and $h_{1}\left(x_{1}, r_{1}, \varepsilon_{1}\right):=h\left(r_{1} x_{1}, r_{1}^{2}, r_{1}^{3} \varepsilon_{1}\right)$. The planes $\left\{r_{1}=0\right\}$ and $\left\{\varepsilon_{1}=0\right\}$ are invariant subspaces of the system (20). Their intersection is the invariant straight line $l_{1}:=\left\{\left(x_{1}, 0,0\right): x_{1} \in \mathbb{R}\right\}$; see Figure 2, Note that $g=O\left(r_{1}\right)$ and $h=O\left(r_{1}\right)$ in the new coordinates. Therefore the dynamics on $l_{1}$ is determined by the equation

$$
x_{1}^{\prime}=-1+x_{1}^{2}
$$




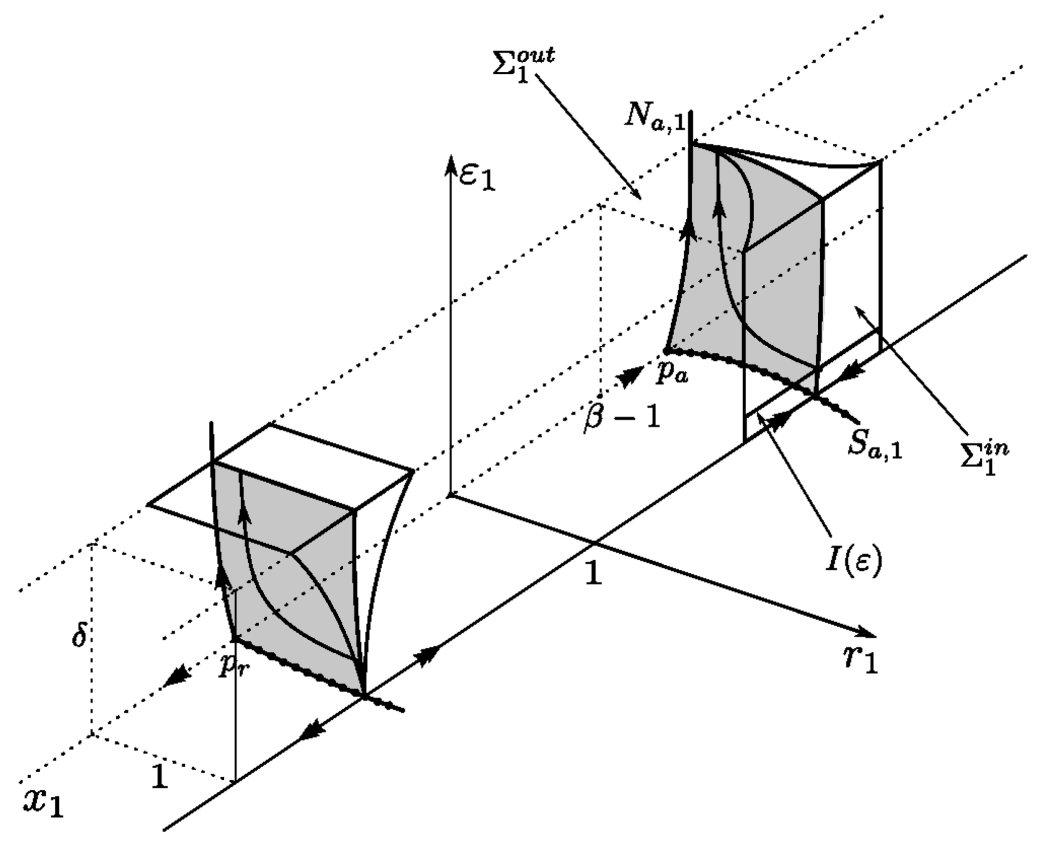

FiguRE 2. Dynamics in the chart $K_{1}$

This equation has two fixed points: $p_{a}:=(-1,0,0)$ and $p_{r}:=(1,0,0)$. The point $p_{a}$ is an attracting hyperbolic point with the eigenvalue -2 ; the point $p_{r}$ is repelling with the eigenvalue 2 (we present this point for clarity; it will not be needed in the analysis).

The dynamics on the invariant plane $\left\{\varepsilon_{1}=0\right\}$ is determined by the system

$$
\left\{\begin{array}{l}
x_{1}^{\prime}=\left(x_{1}^{2}-1\right)(1+h), \\
r_{1}^{\prime}=0 .
\end{array}\right.
$$

This system has the normally hyperbolic curve $S_{a, 1}$ of fixed points $\left(p_{a} \in S_{a, 1}\right)$ and the normally hyperbolic curve $S_{r, 1}\left(p_{r} \in S_{r, 1}\right)$; see Figure 2. In fact, the curves $S_{a, 1}$ and $S_{r, 1}$ are branches of the slow curve $S$ described in $\$ 2$,

On the curve $S_{a, 1}$, the linearization of (21) has one zero eigenvalue: $\lambda_{r}=0$, while the other is negative: $\lambda_{x}=-2(1+h)$. We have a similar situation on $S_{r, 1}$, except that $\lambda_{x}=2(1+h)$.

The dynamics on the invariant plane $\left\{r_{1}=0\right\}$ is determined by the system

$$
\left\{\begin{array}{l}
x_{1}^{\prime}=-1+x_{1}^{2}+\frac{1}{2} \varepsilon_{1} x_{1}, \\
\varepsilon_{1}^{\prime}=\frac{3}{2} \varepsilon_{1}^{2} .
\end{array}\right.
$$

The points $p_{a}$ and $p_{r}$ are fixed points for it.

As already mentioned, we shall only need the point $p_{a}$. At this point, one eigenvalue of the linearization is zero with an eigenvector $(-1,4)$. Consequently, there exists a one-dimensional centre manifold $N_{a, 1}$ of the point $p_{a}$, along which $\varepsilon_{1}$ increases under the action of the flow (for $\varepsilon_{1}>0$ ). It follows from topological considerations that the manifold $N_{a, 1}$ is uniquely determined for $\varepsilon_{1}>0$.

Thus, we have proved the following lemma. 
Lemma 9 (8], Lemma 2.5). The linearization of the system (20) at the point $p_{a}$ has the following eigenvalues:

- $\lambda_{1}=-2$ with an eigenvector $(1,0,0)$ (corresponding to the direction $l_{1}$ );

- $\lambda_{2}=0$ with an eigenvector tangent to $S_{a, 1}$;

- $\lambda_{3}=0$ with an eigenvector $(-1,0,4)$ corresponding to the central direction of the invariant plane $\left\{r_{1}=0\right\}$.

The following assertion follows from the theory of centre manifolds.

Proposition 10. In a sufficiently small neighbourhood of the point $\left(x_{1}, r_{1}, \varepsilon_{1}\right)=$ $(-1,0,0)$, there exists an attracting two-dimensional $C^{k}$-smooth centre manifold $M_{a}$ of the point $p_{a}$ that contains the fixed point curve $S_{a}$ and the centre manifold $N_{a}$. The part of $N_{a}$ corresponding to $\varepsilon_{1}>0$ is uniquely determined.

We introduce the notation $\delta:=e^{-3}$. Recall that $\beta=1 / 12$. We define the transversals

$$
\Sigma_{1}^{\text {in }}:=\left\{\left(x_{1}, r_{1}, \varepsilon_{1}\right) \in D_{1}: r_{1}=1,\left|1+x_{1}\right|<\beta, 0<\varepsilon_{1}<\delta\right\}
$$

and

$$
\Sigma_{1}^{\text {out }}:=\left\{\left(x_{1}, r_{1}, \varepsilon_{1}\right) \in D_{1}: \varepsilon_{1}=\delta,\left|1+x_{1}\right|<\beta, 0<r_{1}<1\right\} .
$$

Remark 11. The transversal $\Sigma^{\text {in }}:=\Delta^{\text {in }} \times\left(0, \varepsilon_{0}\right)$ goes inside the transversal $\Sigma_{1}^{\text {in }}$ under the transformation (13).

It is easy to verify that for $\delta=e^{-3}<1 / 13$ the vector field on the lateral walls of the domain

$$
\left\{\left|1+x_{1}\right|<\beta, 0<r_{1}<1,0<\varepsilon_{1}<\delta\right\}
$$

is directed inside it. Therefore all the trajectories entering this domain through $\Sigma_{1}^{\text {in }}$ go out of it through $\Sigma_{1}^{\text {out }}$.

Proposition 12. Suppose that conditions (10) hold. Then

- the map $\Pi_{1}: \Sigma_{1}^{\text {in }} \rightarrow \Sigma_{1}^{\text {out }}$ exists;

- if $\epsilon<e^{-4}$, then the restriction $P_{1, \epsilon}:=\left.\Pi_{1}\right|_{\varepsilon_{1}=\epsilon}$ is a contraction with coefficient $e^{-1 /(6 \epsilon)}$.

Proof. First of all we use a change of time to 'divide' the system (20) by the expression $\varepsilon_{1}\left(1-g_{1}\right)$ (in the remainder of this proof we shall omit the subscript "1"). As a result the system takes the form

$$
\left\{\begin{array}{l}
x^{\prime}=\left(x^{2}-1\right) \frac{1+h}{\varepsilon(1-g)}+x / 2=: \mathcal{F}(x, r, \varepsilon), \\
r^{\prime}=-r / 2, \\
\varepsilon^{\prime}=3 \varepsilon / 2 .
\end{array}\right.
$$

Consider some initial condition $\left(x_{\mathrm{in}}, r_{\mathrm{in}}, \varepsilon_{\mathrm{in}}\right)$ on the entrance transversal. We now estimate the derivative with respect to $x_{\text {in }}$ of the recurrence map from the entrance transversal to the exit one. In other words, we are interested in the vector

$$
\left(\partial x / \partial x_{\mathrm{in}}, \partial r / \partial x_{\mathrm{in}}, \partial \varepsilon / \partial x_{\mathrm{in}}\right) .
$$

We see from the form of the system (23) that only its first coordinate is nonzero.

The equation in variations for the system (23) gives the following equation for the vector (24):

$$
\frac{d}{d t}\left(\begin{array}{l}
\partial x / \partial x_{\text {in }} \\
\partial r / \partial x_{\text {in }} \\
\partial \varepsilon / \partial x_{\text {in }}
\end{array}\right)=\left(\begin{array}{ccc}
\partial \mathcal{F} / \partial x & \partial \mathcal{F} / \partial r & \partial \mathcal{F} / \partial \varepsilon \\
0 & -1 / 2 & 0 \\
0 & 0 & 3 / 2
\end{array}\right)\left(\begin{array}{l}
\partial x / \partial x_{\text {in }} \\
\partial r / \partial x_{\mathrm{in}} \\
\partial \varepsilon / \partial x_{\mathrm{in}}
\end{array}\right)
$$


It follows from (25) that $\partial r / \partial x_{\mathrm{in}} \equiv \partial \varepsilon / \partial x_{\mathrm{in}} \equiv 0$, and the derivative of the transformation map of the phase flow over time $T$ satisfies

$$
\ln \left(\partial x / \partial x_{\text {in }}\right)=\int_{0}^{T} \mathcal{F}_{x}^{\prime} d t .
$$

Consequently, we need to estimate $\mathcal{F}_{x}^{\prime}$ and the recurrence time $T$ from the entrance transversal to the exit one. We have

$$
\mathcal{F}_{x}^{\prime}=\frac{1}{2}+\frac{1}{\varepsilon}\left[\left(2 x \frac{1+h}{1-g}\right)+\left(\left(x^{2}-1\right) \frac{h_{x}^{\prime}(1-g)+g_{x}^{\prime}(1+h)}{(1-g)^{2}}\right)\right] .
$$

We shall estimate the expression in brackets:

$$
\begin{aligned}
\frac{1}{\varepsilon}\left[2 x \frac{1+h}{1-g}\right. & \left.+\left(x^{2}-1\right) \frac{h_{x}^{\prime}(1-g)+g_{x}^{\prime}(1+h)}{(1-g)^{2}}\right] \\
& \leq \frac{1}{\varepsilon}\left[\frac{2}{3}(-1+\beta)+\max \left|x^{2}-1\right| \cdot \max \left|\frac{h_{x}^{\prime}(1-g)+g_{x}^{\prime}(1+h)}{(1-g)^{2}}\right|\right] \\
& \leq \frac{1}{\varepsilon}\left[-\frac{11}{18}+\beta(\beta+2)\left(\max \left|h_{x}^{\prime}\right|+\max \left|g_{x}^{\prime}\right|\right) \cdot 6\right] \\
& \leq \frac{1}{\varepsilon}\left[-\frac{11}{18}+\frac{1}{48}\right]<\frac{1}{\varepsilon}\left[-\frac{1}{2 e^{3}}-\frac{1}{2}\right] \leq-\frac{1}{2}-\frac{1}{2 \varepsilon} .
\end{aligned}
$$

We have established these inequalities using (9), (10), and the fact that $\varepsilon \leq e^{-3}$ and $\beta=1 / 12$. Hence the recurrence map is a contraction.

To obtain a more precise estimate, we decompose the recurrence map into the composition $P_{1, \varepsilon_{\text {in }}}=P_{\text {out }} \circ P_{\text {in }}$, where the first map is the recurrence map from $\left\{\varepsilon=\varepsilon_{\text {in }}\right\}$ to $\left\{\varepsilon=e \varepsilon_{\text {in }}\right\}$ (this is possible, since $\varepsilon_{\text {in }} \leq e^{-4}=e^{-1} \varepsilon_{\text {out }}$ by hypothesis). It follows from the inequality $\mathcal{F}_{x}^{\prime}<0$ that each of the terms of the composition is nonexpansive.

The recurrence time $T_{1}$ for $P_{\text {in }}$ is equal to

$$
\frac{2}{3}\left(\ln \left(e \varepsilon_{\mathrm{in}}\right)-\ln \varepsilon_{\mathrm{in}}\right)=\frac{2}{3} .
$$

For $P_{\text {in }}$, the estimate for $\mathcal{F}_{x}^{\prime}$ takes the form

$$
\mathcal{F}_{x}^{\prime} \leq-\frac{1}{2 e \varepsilon_{\text {in }}} .
$$

Thus, the derivative of the whole composition is estimated by the expression

$$
\left(\Pi_{1, \varepsilon_{\text {in }}}\right)_{x_{\text {in }}}^{\prime} \leq e^{-1 /\left(2 e \varepsilon_{\text {in }}\right)}<e^{-1 /\left(6 \varepsilon_{\text {in }}\right)} .
$$

\section{$\S 5$. Dynamics in the Chart $K_{2}$ : The map 'Near the JumP'}

The transformation (14) is a simple renormalization of the variables $(x, y)$, since $r_{2}=$ $\varepsilon^{1 / 3}$. Substituting (14) into (11) (see also (6)), we obtain the vector field $\bar{X}$ in the chart $K_{2}\left(r_{2}^{\prime}=\varepsilon^{\prime}=0\right)$ :

$$
\left\{\begin{array}{l}
r_{2} \dot{x}_{2}=F\left(r_{2} x_{2}, r_{2}^{2} y_{2}, r_{2}^{3}\right), \\
\dot{y}_{2}=r_{2} G\left(r_{2} x_{2}, r_{2}^{2} y_{2}, r_{2}^{3}\right), \\
\dot{r}_{2}=0 .
\end{array}\right.
$$

We perform the change of time $t_{2}:=r_{2} t$. Then $\dot{x}=r_{2} x_{2}^{\prime}$, and we obtain

$$
\left\{\begin{array}{l}
x_{2}^{\prime}=\left(x_{2}^{2}-y_{2}\right)\left(1+h\left(r_{2} x_{2}, r_{2}^{2} y_{2}, r_{2}^{3}\right)\right), \\
y_{2}^{\prime}=g\left(r_{2} x_{2}, r_{2}^{2} y_{2}, r_{2}^{3}\right)-1, \\
r_{2}^{\prime}=0 .
\end{array}\right.
$$


We introduce the notation

$$
g_{2}\left(x_{2}, y_{2}, r_{2}\right):=g\left(r_{2} x_{2}, r_{2}^{2} y_{2}, r_{2}^{3}\right)
$$

and

$$
h_{2}\left(x_{2}, y_{2}, r_{2}\right):=h\left(r_{2} x_{2}, r_{2}^{2} y_{2}, r_{2}^{3}\right) .
$$

For $r_{2}=0$ we obtain the Riccati equation:

$$
\left\{\begin{array}{l}
x_{2}^{\prime}=x_{2}^{2}-y_{2} \\
y_{2}^{\prime}=-1
\end{array}\right.
$$

In the following proposition we omit the subscripts to make it easier to read.

Proposition 13 (see [5], pp. 76-81). Equation (31) has the following properties.

(1) Every trajectory has a vertical asymptote $y=y_{r}$ (where $y_{r}$ depends on the trajectory) which this trajectory approaches on the right as $x \rightarrow \infty$.

(2) There exists a unique trajectory $\gamma_{2}$ which can be parametrized in the form $(x, s(x)), x \in \mathbb{R}$. It approaches the upper branch of the parabola $x^{2}-y=0$ as $x \rightarrow-\infty$.

(3) The function $s(x)$ has the asymptotic formulae

$$
\begin{array}{ll}
s(x)=x^{2}+\frac{1}{2 x}+O\left(1 / x^{4}\right), & x \rightarrow-\infty, \\
s(x)=-\Omega_{0}+\frac{1}{x}+O\left(1 / x^{3}\right), & x \rightarrow \infty .
\end{array}
$$

(4) All the trajectories below $\gamma_{2}$ in reverse time approach the lower branch of the parabola $x^{2}-y=0$.

(5) All the trajectories above $\gamma_{2}$ have a vertical asymptote $y=y_{l}>y_{r}$ (where $y_{l}$ depends on the trajectory) which the orbits approach on the left as $x \rightarrow-\infty$.

The assertions of Proposition 13 are illustrated in Figure 3.

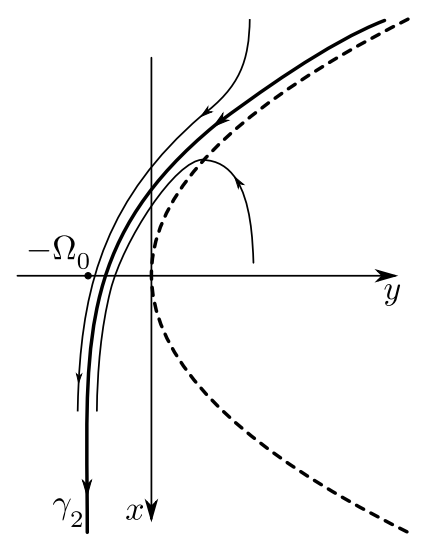

Figure 3. Solutions of equation (31)

Remark 14. The number $\Omega_{0}$ is the smallest positive zero of the equation

$$
J_{-1 / 3}\left(\frac{2 z^{3 / 2}}{3}\right)+J_{1 / 3}\left(\frac{2 z^{3 / 2}}{3}\right)=0,
$$

where the $J_{\star}$ are Bessel functions of the first kind. 
Proposition 15 (8], Proposition 2.6, part 5). The branch $N_{a, 1} \cap\left\{\varepsilon_{1}>0\right\}$ coincides with $\gamma_{1}:=\kappa_{12}^{-1}\left(\gamma_{2}\right)$ wherever $\kappa_{12}$ is defined, that is, for the part of $\gamma_{2}$ with $y_{2}>0$.

Proof. The assertion follows from the uniqueness of $N_{a, 1}$ and the fact that $\kappa_{12}^{-1}\left(\gamma_{2}\right)$ converges to $p_{a}$ (this is a calculation) and is tangent to the direction $(-1,0,4)$ as $x_{2} \rightarrow-\infty$.

Note that under the change of coordinates the exit transversal $\Sigma_{1}^{\text {out }}$ of the chart $K_{1}$ goes to the entrance transversal $\Sigma_{2}^{\text {in }}$ of the chart $K_{2}$ :

$$
\Sigma_{2}^{\text {in }}:=\left\{\left(x_{2}, y_{2}, r_{2}\right): x_{2} \in I_{2}^{\text {in }}, y_{2}=\delta^{-2 / 3}=e^{2}, r_{2} \leq \delta^{1 / 3}=e^{-1}\right\},
$$

where

$$
I_{2}^{\text {in }}\left(\beta_{2}, \delta\right):=\left(-\delta^{-1 / 3}-\beta_{2},-\delta^{-1 / 3}+\beta_{2}\right)=\left(-\frac{13 e}{12},-\frac{11 e}{12}\right), \quad \beta_{2}:=\beta \delta^{-1 / 3}=\frac{e}{12} .
$$

Propositions 10, 12, and 15] imply that the trajectory $\gamma_{2}$ intersects $\Sigma_{2}^{\text {in }}$.

Consider the exit transversal

$$
\Sigma_{2}^{\text {out }}:=\left\{\left(x_{2}, y_{2}, r_{2}\right): x_{2}=\delta^{-1 / 3}=e, y_{2} \in I_{2}^{\text {out }}, r_{2} \leq \delta^{1 / 3}=e^{-1}\right\},
$$

where

$$
I_{2}^{\text {out }}:=(-e, 0)
$$

We will show below that the recurrence map $\Pi_{2}$ takes the transversal $\Sigma_{2}^{\text {in }}$ into the interior of the transversal $\Sigma_{2}^{\text {out }}$. To do this it is sufficient to show that for any $r_{2} \in\left(0, e^{-1}\right)$, the recurrence map $\Pi_{2, r}: \Sigma_{2, r}^{\text {in }} \rightarrow \Sigma_{2, r}^{\text {out }}$ exists for the system

$$
\left\{\begin{array}{l}
x_{2}^{\prime}=\left(x_{2}^{2}-y_{2}\right)\left(1+h_{2}\right) \\
y_{2}^{\prime}=g_{2}-1
\end{array}\right.
$$

(Here $\Sigma_{2, r}^{i}:=\Sigma_{2}^{i} \cap\left\{r_{2}=r\right\}, i=$ in, out.) Moreover, we will estimate the derivative of the map $\Pi_{2, r}$.

Proposition 16. Suppose that conditions (9) and (10) hold. Then the recurrence map $\Pi_{2, r}$

- exists;

- expands no faster than by a factor of $e^{10^{3}}$.

Proof. The scheme of the proof is similar to the proof on the chart $K_{1}$. Using a change of time we reduce the system (32) to the form

$$
\left\{\begin{array}{l}
x^{\prime}=\left(x^{2}-y\right)(1+h) /(1-g)=: \mathcal{F}(x, y, r), \\
y^{\prime}=-1
\end{array}\right.
$$

Then we use the variational equation to find the required estimate.

As in the case already considered above,

$$
\ln \left(\partial x / \partial x_{\mathrm{in}}\right)=\int_{0}^{T} \mathcal{F}_{x}^{\prime} d t
$$

We shall estimate the expression under the integral. We have

$$
\mathcal{F}_{x}^{\prime}=2 x \frac{1+h}{1-g}+\left(x^{2}-y\right) \frac{h_{x}^{\prime}(1-g)+g_{x}^{\prime}(1+h)}{(1-g)^{2}} .
$$

It is easy to see that

$$
|2 x(1+h) /(1-g)| \leq 6|x| \leq \frac{13}{2} e \leq 3 e^{2}
$$


and

$$
\begin{aligned}
\left|\left(x^{2}-y\right) \frac{h_{x}^{\prime}(1-g)+g_{x}^{\prime}(1+h)}{(1-g)^{2}}\right| & \leq 6\left|x^{2}-y\right| \cdot\left(\left|h_{x}^{\prime}\right|+\left|g_{x}^{\prime}\right|\right) \\
& \leq 15 e^{2} \cdot\left(\max \left|h_{x}^{\prime}\right|+\max \left|g_{x}^{\prime}\right|\right) .
\end{aligned}
$$

Hence,

$$
\mathcal{F}_{x}^{\prime} \leq 4 e^{2}
$$

We now need to estimate the recurrence time $T$. It follows from the chain

$$
\left(x^{2}-y\right) \frac{1+h}{1-g} \geq \frac{x^{2}-y}{3} \geq-\frac{y}{3}=\frac{-e^{2}+t}{3}
$$

that it is sufficient to estimate the time in which the trajectory of the equation

$$
x^{\prime}=\frac{-e^{2}+t}{3}
$$

with the initial condition $x_{\text {in }}=-13 e / 12$ intersects the straight line $\{x=e\}$. Obviously, the longest time is required for the trajectory starting from the point with minimal $x$ coordinate. It is easy to verify that this time does not exceed $e^{2}+e$. Thus, we have the estimate

$$
T \leq e^{2}+e<2 e^{2} .
$$

We observe that, together with the estimate for the time $T$, we have proved that the required map does exist. Indeed, we have proved that the trajectories starting from the entrance transversal intersect the straight line $\{x=e\}$, and they do this to the right of the left-hand boundary of the exit transversal. It remains to show that they intersect this line to the left of the right-hand boundary of the exit transversal. It follows from (33) that all the trajectories starting above the $y$-axis inside the parabola leave this domain through the upper branch of the parabola. Thus, these trajectories intersect the $x$-axis to the left of zero. It now follows from the inequality $y^{\prime}<0$ that they can intersect the straight line $\{x=e\}$ only to the left of zero.

Putting (38) and (41) together we obtain an estimate for the derivative of the recurrence transformation at a fixed time:

$$
\partial x / \partial x_{\text {in }} \leq e^{8 e^{4}} .
$$

It remains to estimate the modulus of the projection of the vector $\left(\partial x / \partial x_{\text {in }}, 0,0\right)$ onto the transversal $\Sigma_{2}^{\text {out }}$ along the vector field of the system. To do this we need to multiply the estimate (42) by the following estimate for the expression $y^{\prime} / x^{\prime}$ on the exit transversal:

$$
\left|y^{\prime} / x^{\prime}\right|=\left|\frac{1-g}{\left(x^{2}-y\right)(1+h)}\right| \leq \frac{3}{e^{2}} .
$$

The resulting estimate takes the form

$$
\left(\Pi_{2, r}\right)^{\prime} \leq e^{10^{3}} .
$$

\section{$\S 6$. Dynamics in the Chart $K_{3}$ : The map 'After the Jump'}

After applying the transformation (15) to the system (11) and changing the time $t:=$ $r_{3} \tau$, we obtain the system

$$
\left\{\begin{array}{l}
r_{3}^{\prime}=r_{3}\left(1-y_{3}\right)\left(1+h_{3}\right), \\
y_{3}^{\prime}=\varepsilon_{3} G_{3}-2 y_{3}\left(1-y_{3}\right)\left(1+h_{3}\right), \\
\varepsilon_{3}^{\prime}=-3 \varepsilon_{3}\left(1-y_{3}\right)\left(1+h_{3}\right)
\end{array}\right.
$$


where

$$
h_{3}\left(r_{3}, y_{3}, \varepsilon_{3}\right):=h\left(r_{3}, r_{3}^{2} y_{3}, r_{3}^{3} \varepsilon_{3}\right)
$$

and

$$
G_{3}\left(r_{3}, y_{3}, \varepsilon_{3}\right):=G\left(r_{3}, r_{3}^{2} y_{3}, r_{3}^{3} \varepsilon_{3}\right) .
$$

The planes $\left\{\varepsilon_{3}=0\right\}$ and $\left\{r_{3}=0\right\}$ and the axis $y_{3}$ are invariant under the flow of the system (44).

Lemma 17 ([8], Lemma 2.9). The point $q_{\text {out }}:=(0,0,0)$ is a hyperbolic singular point of the system (44) with the following eigenvalues:

- $\lambda_{1}=1$ with eigenvector $(1,0,0)$ corresponding to the flow on $\left\{\varepsilon_{3}=0\right\}$;

- $\lambda_{2}=-2$ with eigenvector $(0,1,0)$ corresponding to the direction of the $y_{3}$-axis;

- $\lambda_{3}=-3$ with eigenvector $(0,1,1)$ corresponding to the flow on $\left\{r_{3}=0\right\}$.

Proof. This consists of calculations.

Let $\gamma_{3}:=\kappa_{23}\left(\gamma_{2}\right)$.

Lemma 18. The trajectory $\gamma_{3}$ lies in the plane $\left\{r_{3}=0\right\}$, converges to the point $q_{\text {out }}$ as $\varepsilon_{3} \rightarrow 0$, and is tangent to the vector $(0,1,0)$ at this point.

Proof. Part (3) of Proposition 13 and the change of coordinates (15) imply that the trajectory $\gamma_{3}$ has the asymptotic coordinates $\left(0,-\Omega_{0} \varepsilon_{3}^{2 / 3}+\varepsilon_{3}+O\left(\varepsilon_{3}^{5 / 3}\right), \varepsilon_{3}\right)$ as $\varepsilon_{3} \rightarrow 0$.

To describe the flow in a neighbourhood of $q_{\text {out }}$ we define the transversals

$$
\begin{aligned}
\Sigma_{3}^{\text {in }} & =\left\{\left(r_{3}, y_{3}, \varepsilon_{3}\right): r_{3} \in(0,1), y_{3} \in(-1 / 2,0), \varepsilon_{3}=\delta\right\}, \\
\Sigma_{3}^{\text {out }} & =\left\{\left(r_{3}, y_{3}, \varepsilon_{3}\right): r_{3}=1, y_{3} \in(-1 / 2,0), \varepsilon_{3}=(0, \delta)\right\} ;
\end{aligned}
$$

see Figure 4

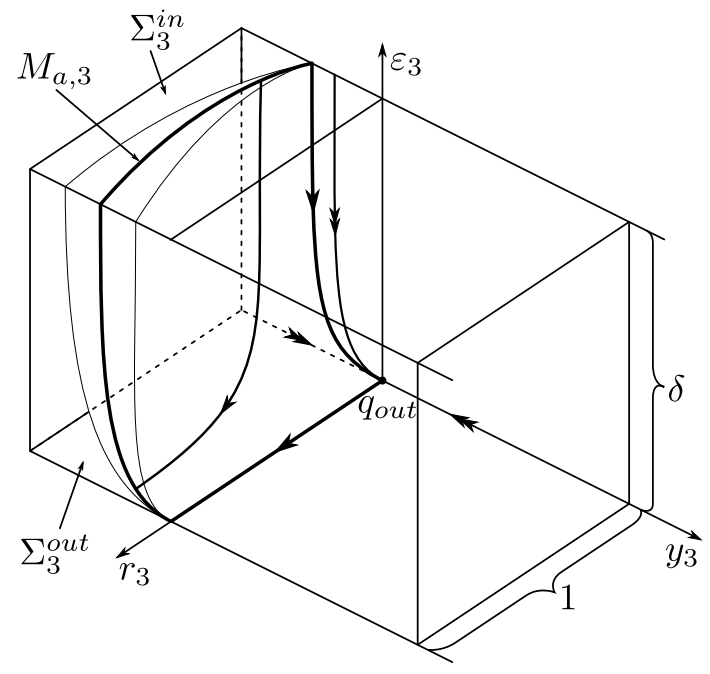

FiguRE 4. A neighbourhood of the point $q_{\text {out }}$ in the chart $K_{3}$

Let $\Pi_{3}: \Sigma_{3}^{\text {in }} \rightarrow \Sigma_{3}^{\text {out }}$ be the recurrence map.

We introduce the notation

$$
D:=e^{-3} \text {. }
$$


Proposition 19. For $y_{3} \in(-1 / 2,0)$ and $r_{3} \leq D$ the recurrence map $\Pi_{3}$ of the system (44) has the form

where

$$
\Pi_{3}\left(r_{3}, y_{3}, \delta\right)=\left(\begin{array}{c}
1 \\
\Pi_{32}\left(r_{3}, y_{3}, \delta\right) \\
r_{3}^{3} \delta
\end{array}\right),
$$

$$
-\frac{3}{2} r_{3}^{2} \leq \Pi_{32}\left(r_{3}, y_{3}, \delta\right) \leq-2^{-6} \delta r_{3}^{2} .
$$

Proof. Our aim is to obtain a formula for the map $\Pi_{3}$. We divide the system (44) by $\left(1-y_{3}\right)\left(1+h_{3}\right)$ (under our assumptions this expression does not vanish):

$$
\left\{\begin{array}{l}
r_{3}^{\prime}=r_{3}, \\
y_{3}^{\prime}=\frac{\varepsilon_{3} G_{3}}{\left(1-y_{3}\right)\left(1+h_{3}\right)}-2 y_{3}=-2 y_{3}-\frac{\varepsilon_{3}}{1-y_{3}}+r_{3} \varepsilon_{3} \mathcal{H}\left(r_{3}, y_{3}, \varepsilon_{3}\right), \\
\varepsilon_{3}^{\prime}=-3 \varepsilon_{3},
\end{array}\right.
$$

where $\mathcal{H}$ is some function.

We introduce the notation

$$
V:=\left\{0<\varepsilon_{3} \leq \delta, y_{3} \in(-1 / 2,0), r_{3} \leq D\right\} .
$$

Lemma 20 (Estimating the nonlinear terms). In the domain $V$ the function $\mathcal{H}$ in formula (47) satisfies the inequality

$$
\left|\mathcal{H}\left(r_{3}, y_{3}, \varepsilon_{3}\right)\right| \leq D_{H},
$$

where $D_{H}=12\left(\max _{U}\|\operatorname{grad} g\|_{1}+\max _{U}\|\operatorname{grad} h\|_{1}\right)$.

This assertion is proved below (see p. 185).

We consider the system (47) for $r_{3}=0$, that is, the system

$$
\left\{\begin{array}{l}
y_{3}^{\prime}=-2 y_{3}-\frac{\varepsilon_{3}}{1-y_{3}}, \\
\varepsilon_{3}^{\prime}=-3 \varepsilon_{3} .
\end{array}\right.
$$

Lemma 21 (Change of coordinates). For $\left|y_{3}\right| \leq 1 / 2$ there exists a change of coordinates of the form

$$
y_{3}=\psi\left(\widetilde{y}_{3}, \varepsilon_{3}\right), \quad \widetilde{y}_{3}=\widetilde{\psi}\left(y_{3}, \varepsilon_{3}\right)
$$

reducing the system (48) to a linear form such that the following estimates hold:

$$
|\widetilde{\psi}-\mathrm{id}| \leq 2\left|y_{3} \varepsilon_{3}\right|
$$

and

$$
|\psi-\mathrm{id}| \leq 2\left|\widetilde{y}_{3} \varepsilon_{3}\right| .
$$

This lemma is proved below (see p. 186).

After making the transformation $y_{3}=\psi\left(\widetilde{y}_{3}, \varepsilon_{3}\right)$, the system (47) turns into the system

$$
\left\{\begin{array}{l}
r_{3}^{\prime}=r_{3}, \\
\widetilde{y}_{3}^{\prime}=-2 \widetilde{y}_{3}-\varepsilon_{3}+r_{3} \varepsilon_{3} H\left(r_{3}, \widetilde{y}_{3}, \varepsilon_{3}\right), \\
\varepsilon_{3}^{\prime}=-3 \varepsilon_{3},
\end{array}\right.
$$

where $H\left(r_{3}, \widetilde{y}_{3}, \varepsilon_{3}\right):=\mathcal{H}\left(r_{3}, \widetilde{\psi}\left(y_{3}, \varepsilon_{3}\right), \varepsilon_{3}\right)$.

Let $\widetilde{V}$ be the image of the domain $V$ under the change of coordinates $\left(r_{3}, y_{3}, \varepsilon_{3}\right) \rightarrow$ $\left(r_{3}, \widetilde{y}_{3}, \varepsilon_{3}\right)$. It follows from the form of $H$ and from Lemma 20 that

$$
\max _{\widetilde{V}}|H| \leq D_{H}
$$


In what follows we omit the subscript 3 from the variables to make it easier to read.

We fix $\left(r_{\text {in }}, \widetilde{y}_{\text {in }}, \delta\right) \in \Sigma^{\text {in }}$ and $\left(1, \widetilde{y}_{\text {out }}, \varepsilon_{\text {out }}\right) \in \Sigma^{\text {out }}$. We consider the solution $(r, \widetilde{y}, \varepsilon)(t)$ of the system (51) and $T>0$ such that

$$
\begin{aligned}
& r(0)=r_{\mathrm{in}}, \quad r(T)=1 ; \\
& \widetilde{y}(0)=\widetilde{y}_{\text {in }}, \quad \widetilde{y}(T)=\widetilde{y}_{\text {out }} ; \\
& \varepsilon(0)=\delta, \quad \varepsilon(T)=\varepsilon_{\text {out }} .
\end{aligned}
$$

The first equation in (51) has the exact solution

$$
r=e^{t} r_{\mathrm{in}}
$$

the requirement $r(T)=1$ gives an explicit formula for $T$ :

$$
T=\ln r_{\text {in }}^{-1} \text {. }
$$

The last equation of the system (51) has the exact solution

$$
\varepsilon=\delta e^{-3 t},
$$

and we obtain

$$
\varepsilon_{\text {out }}=\delta e^{-3 T}=\delta r_{\text {in }}^{3} .
$$

Now suppose that an upper estimate for $\widetilde{y}(T)$ is known and it is negative. Then we can obtain an estimate for $\Pi_{32}$. Indeed, we have the following chain of inequalities (recall that the coordinate $\widetilde{y}$ is negative):

$$
\Pi_{32}(r, y, \delta)=\psi\left(\widetilde{y}(T), r^{3} \delta\right) \leq \widetilde{y}(T)-2 \widetilde{y}(T) r^{3} \delta=\widetilde{y}(T)\left(1-2 r^{3} \delta\right) \leq \frac{1}{2} \widetilde{y}(T) .
$$

A lower bound can be obtained in similar fashion:

$$
\Pi_{32}(r, y, \delta) \geq \frac{3}{2} \widetilde{y}(T) .
$$

In order to estimate $\widetilde{y}(T)$, we define a new coordinate $z$ by the formula

$$
\widetilde{y}=e^{-2 t}\left(\widetilde{y}_{\text {in }}-\delta+z\right)+\delta e^{-3 t} .
$$

Then we have the equation

$$
\widetilde{y}(T)=\left(\left[\widetilde{y}_{\text {in }}-\delta\right]+z+\delta r_{\text {in }}\right) r_{\text {in }}^{2} .
$$

The third summand in the brackets can be bounded above by $\delta / 16$ for $r_{\text {in }}<1 / 16$.

To estimate the first summand we use the inequality

$$
2|\delta y| \leq-\frac{y-\delta}{2} .
$$

This inequality is proved as follows. Note that $2|\delta y|<|y| / 2$. For $y<0$ the inequality $\delta-y>|y|$ holds, giving the estimate we require.

Using the lemma on change of coordinates and inequality (59) we obtain the estimates

$$
\widetilde{y}_{\text {in }}-\delta \leq y+2|\delta y|-\delta<\frac{1}{2}(y-\delta)
$$

and

$$
\widetilde{y}_{\text {in }}-\delta \geq y-2|\delta y|-\delta>\frac{3}{2}(y-\delta) .
$$

To obtain an estimate for $z$ we derive an equation for its derivative with respect to time. Formula (57) implies the equation

$$
-2 \widetilde{y}-\varepsilon+r \varepsilon H(r, \widetilde{y}, \varepsilon)=\widetilde{y}^{\prime}=-2 e^{-2 t}\left(\widetilde{y}_{\text {in }}-\delta+z\right)+e^{-2 t} z^{\prime}-3 \delta e^{-3 t} .
$$

Rearranging the terms gives:

$$
e^{-2 t} z^{\prime}-r \varepsilon H(r, \widetilde{y}, \varepsilon)=2\left[e^{-2 t}\left(\widetilde{y}_{\text {in }}-\delta+z\right)+\delta e^{-3 t}-\widetilde{y}\right]+\left[\delta e^{-3 t}-\varepsilon\right] .
$$


By (54) and (57), the right-hand side of equation (62) is equal to zero. Using equations (52) and (54), we obtain the equation in $z$ :

$$
z^{\prime}=r_{\mathrm{in}} H_{z}\left(z, r_{\mathrm{in}}, \widetilde{y}_{\mathrm{in}}, t\right),
$$

where

$$
H_{z}\left(z, r_{\mathrm{in}}, \widetilde{y}_{\mathrm{in}}, t\right):=\delta H\left(e^{t} r_{\mathrm{in}}, e^{-2 t}\left(\widetilde{y}_{\mathrm{in}}-\delta+z\right)+\delta e^{-3 t}, \delta e^{-3 t}\right) .
$$

We observe that the function $H_{z}$ is bounded in the corresponding domain by the expression $\delta D_{H}$. Using this fact with (53), we obtain

$$
|z(T)| \leq r_{\text {in }} \delta D_{H} T \leq r_{\text {in }} \delta D_{H} \ln \left(1 / r_{\text {in }}\right) .
$$

Since for $0<s<a^{2}$ an inequality of the form $s \ln s>-a, a>0$, certainly holds 1 the right-hand side of inequality (64) does not exceed $\delta / 16$ for $r_{\text {in }} \leq e^{-3}<\left(16 D_{H}\right)^{-2}$. In view of the inequality $\delta / 2 \leq-(y-\delta)$ we obtain the estimate

$$
|z| \leq-\frac{y-\delta}{8}
$$

Collecting together the estimates (58), (60), (61), and (65), as well as $|y|<1 / 2$, we obtain the inequalities

$$
\widetilde{y}(T) \leq\left[\frac{1}{2}(y-\delta)-\frac{y-\delta}{8}+\delta r_{\text {in }}\right] r_{\text {in }}^{2} \leq\left[-\frac{3}{16} \delta+e^{-2} \delta\right] r_{\text {in }}^{2} \leq-\left[2^{-5} \delta\right] r_{\text {in }}^{2}
$$

and

$$
\widetilde{y}(T) \geq\left[3(y-\delta) / 2+(y-\delta) / 8+\delta r_{\text {in }}\right] r_{\text {in }}^{2} \geq[13(y-\delta) / 8] r_{\text {in }}^{2} \geq-r_{\text {in }}^{2} .
$$

Inequality (55) now takes the form

$$
\Pi_{32}\left(r_{\mathrm{in}}, y, \delta\right) \leq-2^{-6} \delta r_{\mathrm{in}}^{2},
$$

and inequality (56) takes the form

$$
\Pi_{32}\left(r_{\mathrm{in}}, y, \delta\right) \geq-\frac{3}{2} r_{\mathrm{in}}^{2} .
$$

The proof is complete.

We will now prove the lemma on estimating the nonlinear terms. For the convenience of the reader we repeat its statement.

Lemma 22 (Estimating the nonlinear terms). In the domain $V$ the function $\mathcal{H}$ in formula (47) satisfies the inequality

$$
\left|\mathcal{H}\left(r_{3}, y_{3}, \varepsilon_{3}\right)\right| \leq D_{H},
$$

where $D_{H}=12\left(\max _{U}\|\operatorname{grad} g\|_{L_{1}}+\max _{U}\|\operatorname{grad} h\|_{L_{1}}\right)$.

Proof. By definition (see equation (47)),

$$
r_{3} \varepsilon_{3} \mathcal{H}\left(r_{3}, y_{3}, \varepsilon_{3}\right)=\frac{\varepsilon_{3} G_{3}}{\left(1-y_{3}\right)\left(1+h_{3}\right)}+\frac{\varepsilon_{3}}{1-y_{3}} .
$$

We introduce the notation

$$
N:=\sum_{0}^{\infty}(-h)^{j}, \quad Y:=\sum_{0}^{\infty} y^{j} .
$$

\footnotetext{
${ }^{1}$ Indeed, let $a<1 / e$. We have $\ln (1 / s)<\sqrt{1 / s}$ as $s \rightarrow 0$. Consequently, $\ln s>-\sqrt{1 / s} \Rightarrow s \ln s>$ $-s \sqrt{1 / s}=-\sqrt{s}$. Thus, the inequality $s \ln s>-a$ certainly holds for $0<s<a^{2}$.
} 
Then we have the chain of equations

$$
\begin{aligned}
r_{3} \varepsilon_{3} \mathcal{H}\left(r_{3}, y_{3}, \varepsilon_{3}\right) & =\frac{\varepsilon_{3} G_{3}}{\left(1-y_{3}\right)\left(1+h_{3}\right)}+\frac{\varepsilon_{3}}{1-y_{3}}=\frac{\varepsilon_{3} G_{3}}{1-y_{3}}\left(1-h_{3} N\right)+\frac{\varepsilon_{3}}{1-y_{3}} \\
& =\varepsilon_{3} \frac{g_{3}-h_{3} N+g_{2} h_{3} N}{1-y_{3}}=\varepsilon_{3}\left[g_{3}-h_{3} N+g_{2} h_{3} N\right]\left(1+y_{3} Y\right) .
\end{aligned}
$$

It remains to show that we can take a factor $r_{3}$ out of the brackets and estimate the remaining expression. The possibility of taking out this factor from the functions $g_{3}$ and $h_{3}$ follows from their form and the formulae for the blow-up transformation (15). It is easy to see that the estimates $\left|1+y_{3} Y\right| \leq 2$ and $|N| \leq 2$ hold in the corresponding domain $V$. The chain (68) now implies that

$$
r_{3} \varepsilon_{3} \mathcal{H}\left(r_{3}, y_{3}, \varepsilon_{3}\right) \leq 2 \varepsilon\left(2\left|h_{3}\right|+2\left|g_{3}\right|\right)=4 \varepsilon\left(\left|h_{3}\right|+\left|g_{3}\right|\right) .
$$

We now estimate the expression for $g_{3}$ :

$$
\begin{aligned}
\left|\frac{g_{3}\left(r_{3}, r_{3}^{2} y_{3}, r_{3}^{3} \varepsilon_{3}\right)}{r_{3}}\right| & \leq \max _{V}\left|\frac{d g_{3}}{d r_{3}}\right| \\
& \leq \max _{U}\left(\left|\frac{\partial g}{\partial x}\right|+2\left|\frac{\partial g}{\partial y}\right|+3\left|\frac{\partial g}{\partial \varepsilon}\right|\right) \leq 3 \max _{U}\|\operatorname{grad} g\|_{1},
\end{aligned}
$$

where $\|\operatorname{grad} g\|_{1}:=\left|\frac{\partial g}{\partial x}\right|+\left|\frac{\partial g}{\partial y}\right|+\left|\frac{\partial g}{\partial \varepsilon}\right|$. An estimate for the function $h_{3}$ is obtained in exactly the same way. By collecting all the estimates together we obtain the inequality

$$
|\mathcal{H}| \leq 12\left(\max _{U}\|\operatorname{grad} g\|_{1}+\max _{U}\|\operatorname{grad} h\|_{1}\right) .
$$

The lemma on estimating the nonlinear terms is proved.

We now prove the lemma on change of coordinates. For the convenience of the reader we also repeat its statement.

Lemma 23 (Change of coordinates). For $\left|y_{3}\right| \leq 1 / 2$ there exists a change of coordinates of the form

reducing the system

$$
y_{3}=\psi\left(\widetilde{y}_{3}, \varepsilon_{3}\right), \quad \widetilde{y}_{3}=\widetilde{\psi}\left(y_{3}, \varepsilon_{3}\right)
$$

$$
\left\{\begin{array}{l}
y_{3}^{\prime}=-2 y_{3}-\frac{\varepsilon_{3}}{1-y_{3}}, \\
\varepsilon_{3}^{\prime}=-3 \varepsilon_{3}
\end{array}\right.
$$

to a linear form such that the following estimates hold:

$$
|\widetilde{\psi}-\mathrm{id}| \leq 2\left|y_{3} \varepsilon_{3}\right|
$$

and

$$
|\psi-\mathrm{id}| \leq 2\left|\widetilde{y}_{3} \varepsilon_{3}\right|
$$

Proof. We consider the auxiliary system

$$
\left\{\begin{array}{l}
y^{\prime}=-2 y-\varepsilon y \sum_{j=0}^{\infty} y^{j}, \\
\varepsilon^{\prime}=-3 \varepsilon .
\end{array}\right.
$$

This system differs from the original one because there is no term $-\varepsilon$ in the first equation.

We seek a change of coordinates $y=\psi(z, \varepsilon)$ that reduces the system (75) to a linear form. Note that the same change of coordinates will reduce the system (72) to a linear form. 
We seek it by the standard method, at the $k$ th step 'killing' all nonlinear terms of order $k$. Here the resulting change of coordinates must have the form

$$
y=\psi(z, \varepsilon)=z+\sum_{2}^{\infty} P_{k}(z, \varepsilon), \quad P_{k}:=\sum_{i+j=k} K_{i j} z^{i} \varepsilon^{j} .
$$

Let $\lambda_{y}$ and $\lambda_{\varepsilon}$ be the eigenvalues of the linearization matrix of the system (75). Then $P_{k}$ satisfies the equation

$$
\lambda_{y} P_{k}-\lambda_{y} \frac{\partial P_{k}}{\partial y} y-\lambda_{\varepsilon} \frac{\partial P_{k}}{\partial \varepsilon} \varepsilon=-g_{i j}
$$

(see, for example, [3], equation (3.3.7)). In our case, $g_{i 1}=-\varepsilon y^{i}$, and this equation takes the form

$$
-2 \sum_{i+j=k} K_{i j} z^{i} \varepsilon^{j}+2 \sum_{i+j=k} i K_{i j} z^{i} \varepsilon^{j}+3 \sum_{i+j=k} j K_{i j} z^{i} \varepsilon^{j}=\varepsilon z^{k-1},
$$

whence it is clear that $K_{i j}=0$ for $j \geq 2$.

Introducing the notation $K_{s}:=K_{i 1}, s=k-1$, on $K_{s}$ we obtain:

$$
-2 K_{s}+2 s K_{s}+3 K_{s}=1 \text {. }
$$

Consequently,

$$
K_{s}=\frac{1}{1+2 s} .
$$

As a result, the change of coordinates takes the form

$$
y=z+\sum_{s=1}^{\infty} K_{s} \varepsilon z^{s}=z+\varepsilon z \sum_{0}^{\infty} \frac{z^{s}}{1+2 s} .
$$

Thus, we have constructed the required change of coordinates (the convergence of the series in the required domain is obvious). We now find the requisite estimates. The equation

$$
\psi-\mathrm{id}=\varepsilon z \sum_{0}^{\infty} \frac{z^{s}}{1+2 s}
$$

implies inequality (74):

$$
|\psi-\mathrm{id}| \leq|\varepsilon z| \cdot \sum_{0}^{\infty} \frac{\left|z^{s}\right|}{1+2 s} \leq|\varepsilon z| \cdot \sum_{0}^{\infty}\left|z^{s}\right| \leq \frac{3}{2}|z \varepsilon| .
$$

We now show that inequality (73) is true. Consider some constant $C>0$ and suppose that

$$
|\widetilde{\psi}-\mathrm{id}|>C|y \varepsilon|
$$

Then the following chain of inequalities holds in the domain $U$ :

$$
\frac{3}{2}|z \varepsilon| \geq|y-z|>C|y \varepsilon|=C|\psi(z, \varepsilon)||\varepsilon| \geq C\left|z-\frac{3}{2} z \varepsilon\right||\varepsilon| \geq \frac{5 C}{6}|z||\varepsilon| .
$$

Consequently, inequality (76) cannot hold for $C \geq 9 / 5$. The lemma on change of coordinates is proved.

To prove Theorem 4 we need the following proposition. 
Proposition 24. Let $y_{\text {in }}=\mu\left(r_{\text {in }}\right)$ be a smooth curve on $\Sigma_{3}^{\text {in }}$ defined in a (half-)neighbourhood of zerd 2 such that $\mu(0)<0$.

Then the image of this curve under the action of the map $\Pi_{3}$ on $\Sigma_{3}^{\text {out }}$ can be represented in the form of the graph of $\varepsilon_{\text {out }}=\eta\left(y_{\text {out }}\right)$, where the function $\eta$ is $C^{1}$-smooth and has a one-sided derivative at zero: $\eta^{\prime}(0)=0$.

Proof. As above, we denote objects on the transversal $\Sigma_{3}^{\text {in }}$ by the subscript ${ }_{\text {in }}$, and objects on $\Sigma_{3}^{\text {out }}$ by the subscript out. For coordinates on $\Sigma_{3}^{\text {out }}$ we use $\left(\widetilde{y}_{\text {out }}, \epsilon\right)$, where $\epsilon=\varepsilon_{\text {out }} / \delta=$ $r_{\text {in }}^{3}$.

The idea of the proof is simple: we show that the expression $\eta^{\prime}\left(\widetilde{y}_{\text {out }}\right)=\frac{1}{\partial \widetilde{y}(T) / \partial \epsilon}$ tends to zero as $\widetilde{y}_{\text {out }} \rightarrow 0^{-}$or, which is the same, as $\epsilon \rightarrow 0^{+}$.

Relation (58) implies the equation

$$
\widetilde{y}(T)=\widetilde{y}_{\text {out }}=\left[\widetilde{y}_{\text {in }}-\delta\right] \epsilon^{2 / 3}+\left(z+\delta \epsilon^{1 / 3}\right) \epsilon^{2 / 3}=\left[\mu\left(\epsilon^{1 / 3}\right)-\delta\right] \epsilon^{2 / 3}+\delta \epsilon+z \epsilon^{2 / 3} .
$$

Hence we have the chain

$$
\begin{aligned}
\frac{\partial}{\partial \epsilon} \widetilde{y}(T) & =\delta+\frac{2}{3}\left[\mu\left(\epsilon^{1 / 3}\right)-\delta+z\right] \epsilon^{-1 / 3}+\epsilon^{2 / 3}\left[\frac{\partial}{\partial \epsilon} z+\frac{1}{3} \mu^{\prime}\left(\epsilon^{1 / 3}\right) \epsilon^{-2 / 3}\right] \\
& =\left[\delta+\frac{1}{3} \mu^{\prime}\left(\epsilon^{1 / 3}\right)\right]+\frac{2}{3}\left[\mu\left(\epsilon^{1 / 3}\right)-\delta+z\right] \epsilon^{-1 / 3}+\left[\epsilon^{2 / 3} \frac{\partial}{\partial \epsilon} z(T)\right] .
\end{aligned}
$$

The first summand tends to some constant as $\epsilon \rightarrow 0$, and the second summand tends to an expression of the form $C \cdot \epsilon^{-1 / 3}$. We must analyse the third summand. To do this we observe that

$$
z=z\left(t, \widetilde{y}_{\text {in }}\right)=z\left(t, \mu\left(\epsilon^{1 / 3}\right)\right) .
$$

Since the relation $T=-\frac{1}{3} \ln \epsilon$ holds on $\Sigma_{3}^{\text {out }}$, we have

$$
\begin{aligned}
\frac{\partial}{\partial \epsilon} z(T)=\frac{\partial z}{\partial t} \frac{\partial T}{\partial \epsilon}+\frac{\partial z}{\partial \widetilde{y}} \frac{\partial \widetilde{y}}{\partial \epsilon} & =\left.\dot{z}\right|_{t=T} \frac{\partial T}{\partial \epsilon}+\frac{1}{3} \mu^{\prime}\left(\epsilon^{1 / 3}\right) \epsilon^{-2 / 3} \partial z / \partial \widetilde{y}_{\text {in }} \\
& =-\frac{\delta}{3} \epsilon^{-2 / 3} H(1, \widetilde{y}(T), \delta \epsilon)+\frac{1}{3} \mu^{\prime}\left(\epsilon^{1 / 3}\right) \epsilon^{-2 / 3}
\end{aligned}
$$

We see that the third summand on the right-hand side of the chain (78) also tends to some constant as $\epsilon \rightarrow 0$.

Now the chain (78) implies the required assertion.

\section{$\S 7$. The completion of the proof}

In this section we complete the proof of Theorem 6 , putting the results obtained on the different charts together.

The main idea is to analyse the evolution of the centre manifold $M_{a, 1}$ and the rectangle

$$
R=\left\{\left(x_{1}, 1, \varepsilon_{1}\right): \varepsilon_{1} \in[0, \delta],\left|-1+x_{1}\right| \leq \beta_{1}\right\}
$$

under the action of the flow of the blown-up vector field $\bar{X}$.

The dynamics of the blown-up field $\bar{X}$ is shown in Figure 5 .

Suppose that the hypotheses of the theorem hold.

\footnotetext{
${ }^{2}$ The smoothness at zero is one-sided.
} 


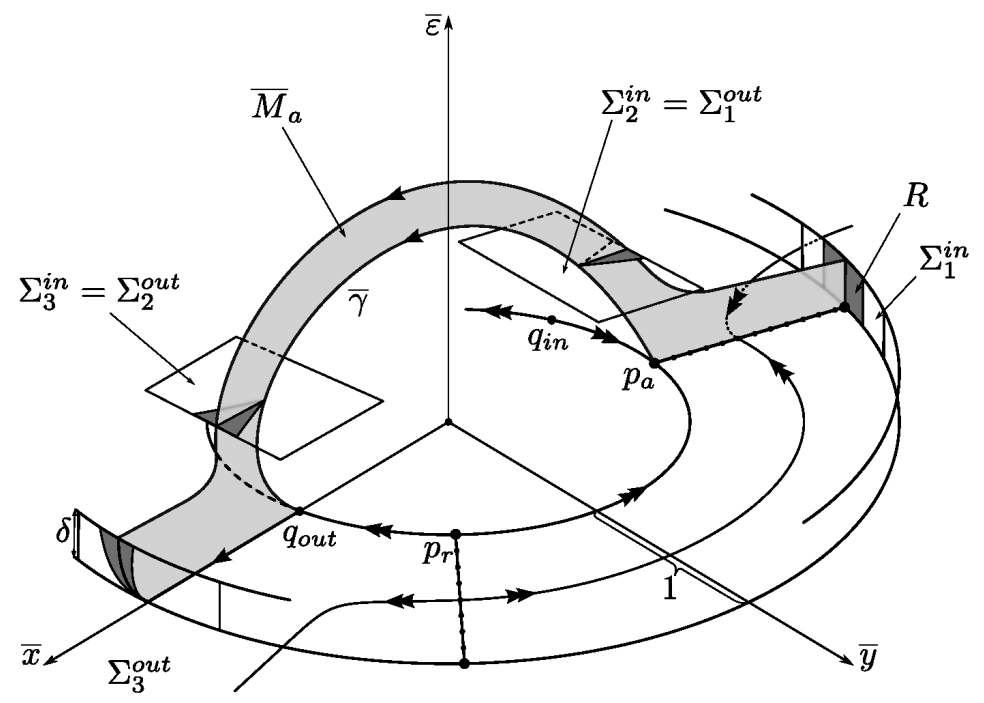

Figure 5. The dynamics of the blown-up vector field

7.1. Proof of existence. We consider the entrance transversal $\Sigma^{\text {in }}$ of the extended system (11):

$$
\Sigma^{\text {in }}:=\left\{\begin{array}{l}
x \in(-1-\beta,-1+\beta), \\
y=1, \\
\varepsilon \in\left(0, \varepsilon_{0}\right) .
\end{array}\right.
$$

We also consider the exit transversal $\Sigma^{\text {out }}$ of the extended system (11):

$$
\Sigma^{\text {out }}:=\left\{\begin{array}{l}
x=1 \\
y \in(-1,0) \\
\varepsilon \in\left(0, \varepsilon_{0}\right)
\end{array}\right.
$$

We claim that the recurrence map $\pi: \Sigma^{\text {in }} \rightarrow \Sigma^{\text {out }}$ exists.

Under the transformation of coordinates (13), the transversal $\Sigma^{\text {in }}$ goes to the transversal

$$
\Sigma_{1}^{\text {in }}=\left\{\begin{array}{l}
x_{1} \in I_{1}^{\text {in }}:=\left(-1-\beta_{1},-1+\beta_{1}\right) \\
r_{1}=1 \\
\varepsilon_{1} \leq \delta
\end{array}\right.
$$

By Proposition 12, the map $\Pi_{1}: \Sigma_{1}^{\text {in }} \rightarrow \Sigma_{1}^{\text {out }}$ is defined, where

$$
\Sigma_{1}^{\text {out }}=\left\{\begin{array}{l}
x_{1} \in I_{1}^{\text {out }}:=I_{1}^{\text {in }}, \\
r_{1} \leq 1 \\
\varepsilon_{1}=\delta
\end{array}\right.
$$

Next, under the change of coordinates (16), the transversal $\Sigma_{1}^{\text {out }}$ goes to the transversal

$$
\Sigma_{2}^{\text {in }}=\left\{\begin{array}{l}
x_{2}=x_{1} \varepsilon_{1}^{-1 / 3} \in I_{2}^{\text {in }}:=\left(-\delta^{-1 / 3}-\delta^{-1 / 3} \beta,-\delta^{-1 / 3}+\delta^{-1 / 3} \beta\right) \\
y_{2}=\varepsilon_{1}^{-2 / 3}=\delta^{-2 / 3} \\
r_{2}=r_{1} \varepsilon_{1}^{1 / 3} \leq \delta^{1 / 3}
\end{array}\right.
$$


By Proposition [16] the map $\Pi_{2}: \Sigma_{2}^{\text {in }} \rightarrow \Sigma_{2}^{\text {out }}$ is defined, where

$$
\Sigma_{2}^{\text {out }}=\left\{\begin{array}{l}
x_{2}=\delta^{-1 / 3}, \\
y_{2} \in I_{2}^{\text {out }}:=(-e, 0), \\
r_{2} \leq \delta^{1 / 3} .
\end{array}\right.
$$

Next, under the change of coordinates (18), the transversal $\Sigma_{2}^{\text {out }}$ goes to some transversal $\Sigma_{3}^{\prime}$ of the chart $K_{3}$. It is easy to verify that $\Sigma_{3}^{\prime} \subset \Sigma_{3}^{\text {in }}$, where

$$
\Sigma_{3}^{\text {in }}=\left\{\begin{array}{l}
\varepsilon_{3}=\delta \\
y_{3} \in I_{3}^{\text {in }}:=(-1 / 2,0), \\
r_{3} \leq 1
\end{array}\right.
$$

By Proposition 19, for $r_{3} \leq D$ the map $\Pi_{3}: \Sigma_{3}^{\text {in }} \rightarrow \Sigma_{3}^{\text {out }}$ is defined, where

$$
\Sigma_{3}^{\text {out }}=\left\{\begin{array}{l}
r_{3}=1, \\
y_{3} \in(-1 / 2,0), \\
\varepsilon_{3} \leq \delta .
\end{array}\right.
$$

In the coordinates $(x, y, \varepsilon)$ the requirement on $r_{3}$ takes the form

$$
\varepsilon \leq \varepsilon_{0}:=\delta D^{3}=e^{-12} .
$$

This follows from the transition formulae (15) and the equations $\varepsilon_{3, \text { out }}=\delta r_{3 \text {,in }}^{3}$ and $r_{3, \text { out }}=1$.

Finally, under the transformation of coordinates (15) the transversal $\Sigma_{3}^{\text {out }}$ goes to the transversal $\Sigma^{\text {out }}$.

Part (11) of Theorem 6 is proved.

7.2. The estimate for the coordinate and the proof of Theorem 4, The map $\pi: \Sigma^{\text {in }} \rightarrow \Sigma^{\text {out }}$ is defined by the following composition:

$$
\pi:=\Phi \circ \Pi_{3} \circ \kappa_{23} \circ \Pi_{2} \circ \kappa_{12} \circ \Pi_{1} \circ \Phi^{-1} .
$$

It is easy to verify that $\kappa_{23} \circ \Pi_{2} \circ \kappa_{12} \circ \Pi_{1}\left(R \cap M_{a, 1}\right)$ is a smooth curve on the transversal $\Sigma_{3}^{\text {in }}$ and this curve is transverse to the plane $\left\{r_{3}=0\right\}$.

It follows from Proposition 19 that $\Pi_{3} \circ \kappa_{23} \circ \Pi_{2} \circ \kappa_{12} \circ \Pi_{1}\left(R \cap M_{a, 1}\right)$ is a curve of the form $y_{3}=\nu_{3}\left(\varepsilon_{3}\right)$, and the following estimates hold: $-2 r_{3}^{2} \leq \nu_{3} r_{3}^{3} \delta \leq-2^{-6} \delta r_{3}^{2}$ or, equivalently,

$$
-2 e^{2} \cdot \varepsilon_{3}^{2 / 3} \leq \nu_{3}\left(\varepsilon_{3}\right) \leq-2^{-6} e^{-1} \cdot \varepsilon_{3}^{2 / 3} .
$$

Finally, under the action of the change of coordinates (15) the curve $y_{3}=\nu_{3}\left(\varepsilon_{3}\right)$ goes to a curve $\nu(\varepsilon)$ such that

$$
-2 e^{2} \cdot \varepsilon^{2 / 3} \leq \nu(\varepsilon) \leq-2^{-6} e^{-1} \cdot \varepsilon^{2 / 3} .
$$

The proof of part (2) of Theorem 6 is complete.

Furthermore, Proposition 24]implies the (one-sided) smoothness of the function $\eta(\varepsilon):=$ $\nu^{-1}$ at zero, which proves Theorem 4. 
7.3. The estimate for the contraction. Since $\varepsilon=\varepsilon_{1}=\varepsilon_{3}$ is a constant 3 of the motion of the flow $\bar{X}$, the lines $\left\{\varepsilon_{1}=\varepsilon=\mathrm{const}\right\}$ on $\Sigma_{1}^{\text {in }}$ are mapped to the lines $\left\{\varepsilon_{3}=\varepsilon=\mathrm{const}\right\}$ on $\Sigma_{3}^{\text {out }}$.

It follows from formulae (13) that the transition from $\Sigma^{\text {in }}$ to $\Sigma_{1}^{\text {in }}$ is nonexpansive. It follows from Proposition 12 that the map $\Pi_{1}$ contracts by a factor of at least $e^{-1 /\left(6 \varepsilon_{1}\right)}=$ $e^{-1 /(6 \varepsilon)}$. It follows from formulae (16) that the transition from $\Sigma_{1}^{\text {out }}$ to $\Sigma_{2}^{\text {in }}$ expands by a factor $\delta^{-1 / 3}$. It follows from Proposition [16 that the map $\Pi_{2}$ expands by a factor of at most $e^{10^{3}}$. It follows from formulae (18) that the transition from $\Sigma_{2}^{\text {out }}$ to $\Sigma_{3}^{\text {in }}$ contracts by $\delta^{2 / 3}$. It follows from inequalities (46) that the map $\Pi_{3}$ is nonexpansive. Finally, it follows from formulae (15) that the transition from the transversal $\Sigma_{3}^{\text {out }}$ to the transversal $\Sigma^{\text {out }}$ is nonexpansive.

Multiplying these coefficients, we obtain the contraction constant: $e^{10^{3}} e^{-1 /(6 \varepsilon)}$. Part (3) of Theorem [6 is proved.

\section{REFERENCES}

[1] V. I. Arnol'd, V. S. Afraĭmovich, Yu. S. Il'yashenko, and L. P. Shil'nikov, Bifurcation theory, Current Problems in Mathematics. Fundamental Directions, vol. 5, 5-218, Itogi Nauki i Tekhniki, VINITI, Moscow, 1986; English transl., Bifurcation theory and catastrophe theory, Dynamical Systems, vol. V, Encyclopaedia Math. Sci., vol. 5, Springer, Berlin, 1994. MR895653

[2] S.-N. Chow, C. Z. Li, and D. Wang, Normal forms and bifurcation of planar vector fields, Cambridge Univ. Press, Cambridge, 1994. MR 1290117 (95i:58161)

[3] J. Guckenheimer and P. Holmes, Nonlinear oscillations, dynamical systems, and bifurcations of vector fields, Appl. Math. Sci., vol. 42, Springer-Verlag, New York, 1990. MR,1139515 (93e:58046)

[4] E. F. Mishchenko and L. S. Pontryagin, Deriving some asymptotic estimates for solutions of differential equations with a small parameter at derivatives, Izv. Akad. Nauk SSSR Ser. Mat. 23 (1959), no. 5, 643-660. (Russian) MR0118916 (22:9685)

[5] E. F. Mishchenko and N. Kh. Rozov, Differential equations with small parameters and relaxation oscillations, Nauka, Moscow, 1975; English transl., Mathematical Concepts and Methods in Science and Engineering, vol. 13, Plenum Press, New York, 1980. MR750298 (85j:34001)

[6] F. Dumortier and R. Roussarie, Canard cycles and center manifolds, Mem. Amer. Math. Soc., vol. 577, Amer. Math. Soc., Providence, RI, 1996. MR.1327208 (96k:34113)

[7] N. Fenichel, Geometric singular perturbation theory for ordinary differential equation, J. Differential Equations, 31 (1979), 53-98. MR524817 (80m:58032)

[8] M. Krupa and P. Szmolyan, Extending geometric singular perturbation theory to nonhyperbolic points - fold and canard points in two dimensions, SIAM J. Math. Anal. 33 (2001), no. 2, 286314. MR.1857972 (2002g:34117)

Research and Development Institute of Power Engineering, Moscow, Russia

E-mail address: pkaleda@yandex.ru

Translated by E. KHUKHRO

\footnotetext{
${ }^{3}$ We are speaking about the transversals $\Sigma_{1}^{\text {in }}$ and $\Sigma_{3}^{\text {out }}$, on which $r_{i}=1$.
} 